\title{
A SIMPLIFIED AND RATIONAL METHOD OF CASTING GOLD INLAYS.
}

\author{
By R. H. Volland, D. D. S., M. D., lowa City, Iowa. \\ Professor of Operative Technic and Dental Pathology State Univorsity of lowa.
}

A $\mathrm{N}$ ACCURATELY fitting gold inlay can be made. In order to obtain such an inlay the several steps in technic must be carried out with extreme care. A well prepared cavity with smooth walls and margins that will allow the shedding of a perfect wax pattern without distortion is the first prerequisite. The making of a wax pattern is an exacting piece of technic and must be well done. The treatment of the wax pattern and the final production of a smooth accurately fitting gold inlay is the object of this paper.

Let it be supposed that a perfect wax model has been made and is mounted on a sprue wire. A wax pattern should be invested as soon as possible after removal from the cavity so as to prevent any possible changes in form due to variation in temperature or to accident. A reasonable sized sprue wire is all that is necessary. A victrola needle has been used with complete satisfaction. However, the wire should be of a length that will permit the investing of the model about half way between the end of the ring and the apex of the former. A deep hole in the former and this hole lined with bee's wax will facilitate this adjustment.

A former should be used to insure uniformly good results. 'The former should provide a positive seating for the ring. Rings of various sizes are recommended but it seems best to use a ring of liberal size. The ring for inlay work should not be less than one inch in diameter. The length of the ring will be determined to a large degree by the height of the cone that forms the crucible. A good working rule is to have approximately as much investment from the apex of the cone and from the end of the ring to the wax pattern as there is on any cross radius from the wax pattern to the inside of the ring. Therefore, if the crucible former is high, a long ring is necessary. 'The reason assigned for the above specifications is that a sufficient amount of investment should surround the mould to prevent surface or superficial changes of the investment, when on heat, from affecting the walls of the mould. This is especially true if the gold is to be melted in the crucible-as in casting with compressed air, gas or steam. A larger ring -one and one-fourth inches in diameter-is needed for this type of apparatus than for a centrifugal machine where the gold is melted outside the crucible. Many different types of rings have been advocated-thin, thick, iron, brass, bronze, perforated, partitioned, etc.,-but the ring is only a tool for moulding the investment to a convenient form. Perfectly fitting gold inlays can be made without the use of a ring during the heating and casting of the gold. This can be easily demonstrated if a centrifugal machine is used. It is true that a ring is more or less necessary to help complete the adaptation of the plunger in a compressed air, gas, or steam apparatus, but the ring itself has nothing to 
do with the fit or misfit of an inlay when the casting is made. A sufficiently large number of experiments has been made by the writer, using a divided ring during the investing but removing it at the time the former and sprue were removed preparatory to putting the case on heat, to substantiate the above statements.

\section{Treatment of the Wax Pattern.}

The sprue wire holding the wax form should be placed in the former and adjusted to proper height. The pattern should then be thoroly washed with grain alcohol applied with a small sable brush. The bristles of this brush should be at least one-half inch long and a tuft of at least one-eighth inch in diameter. Such a brush is soft and pliable and in washing the wax pattern there is little danger of bending or distorting any delicate margin of the wax. The washing of the wax model with alcohol removes any oleaginous material and facilitates the flowing of the investment material closely over the pattern.

\section{Investment Materials.}

Many different investment material formula have been devised and exploited by dentists and manufacturers. In so far as the writer knows the principal materials used in these formula are plaster of Paris, silex, graphite, etc. Each producer claims to have made a combination of materials that will not distort or disintegrate when heated and therefore help to insure a final well fitting casting. The plaster of Paris is used as a binder for the other ingredients just as cement is used as a hinder for the rock and gravel in concrete. Silex is an infusible material and is added to help control distortion of the investment when it is on heat to dissipate or "burn out" the wax pattern. Graphite is added to give smoothness to the walls of the mould when the wax has been dissipated. It is also thought to assist in controlling distortion and in facilitating the adaption of the molten gold to the walls of the mould. This idea, that the presence of graphite, silex or any other material is necessary to control distortion or provide a smooth wall in the mould, is poorly founded. Perfectly smooth and accurately fitting castings are being made by the writer with plaster of Paris alone as the investing material. He has experimented with several brands and formula of investment materials and finds that no smoother or more accurately fitting castings can be made by their use than by the use of plaster of Paris when correctly handled. These findings are not strange when one remembers that the binder of all investments is plaster of Paris. A concrete block will not exhibit its maximum resistance and strength when the binder has been partially or wholly destroyed. An investment loses its desired qualities in proportion to the deterioration of the binder. It is the plaster of Paris of an inlay investment that should receive first consideration and the technic which will be outlined in the following paragraphs will conserve the integrity of the plaster of Paris whether it be used alone as an investment or as a binder of other materials.

\section{InVESTING.}

All materials entering into an investment should be the samc temperature as the wax pattern-namely room temperature.

Plaster of Paris-Ordinary good grade medium setting model plaster is suitable with water at room temperature--the plaster should be mixed to a consistency which will allow the following technic to be completed. The wax pattern is given a thoro painting of this mixture, using a sable brush such as was used to wash the pattern. Care should be taken to see that the plaster goes into all irregularities and gives an even coating. This 
coating will be very thin. When every particle of surface has been reached by this first painting more plaster can be quickly applied with the brush. The ring is now placed in position and the investment completed either by pouring the plaster directly from the bowl or by carrying it to the ring on the spatula. In either case the plaster should be poured down the inside of the ring on one side. It will flow down the side of the ring over the former, rising over the cone, enveloping the sprue and the wax pattern and finally filling the ring. The plaster of Paris should not be assisted to flow by jarring or tapping. After the investment has reached the wax pattern there is always danger of bringing any air bubbles which may be confined in the mixture up to the wax pattern if any jarring or vibrating is resorted to. These air bubbles which fasten themselves to the wax pattern cause corresponding beads of gold on the casting. If the operator keeps in mind the rapidity with which the plaster of Paris sets and the amount of technic that must be accomplished from the time the mixture is made until the ring is full, he can, with a very little experimenting, determine to what consistency the plaster must be mixed. When the ring is rounded full the invested case should be permitted to stand undisturbed until the plaster has set so that it offers considerable resistence to the finger nail. The surplus plaster should be cut even with the end of the ring and the former and sprue removed. The case should immediately be placed on heat, crucible side down.

Other Investments-The manufacturer or dentist who offers a certain investment to the profession has usually worked out a set of rules for the mixing and applying of that particular product and it may be well to follow such instructions, providing the above outlined technic for investing can be followed.

\section{Disstpating the Wax Pattern.}

This process is commonly called "burning out the wax" which is rather misleading. The dissipation of a wax from is accomplished in three ways. (1) Absorption of the molten wax by the investment. (2) Running of the molten wax out into the crucible thru the sprue hole (when the investment is placed on heat with the crucible end down) and (3) Volatilization of the wax. In the treatment of an invested case these three phases work together. They can be accomplished at a temperature that will not materially effect the desirable qualities of a wholly plaster of Paris investment or the plaster of Paris in a combination investment. As soon as the wax begins to melt the porosity of the investment allows the absorption of a portion of it. The absorption of the melted wax. is the greatest factor in dissipating it. The sprue hole offers a gate way thru which some of the molten wax may flow. As more heat is applied some of the more volatile elements of the wax pass away as gases. This should be a very minor part of the process as it requires too much heat. The gases developed in volatilization often ignite and burn with a small flame at the sprue hole. The degree of heat that is reached in this latter treatment of a mould is not only unnecessary but detrimental to the quality of the mould.

Plaster of Paris will withstand a dry even heat of $320^{\circ} \mathrm{F}$, without deterioration of its desirable qualities as an investment. $300^{\circ} \mathrm{F}$ is sufficient to dissipate the wax provided it is subjected to that heat long enough. Wax patterns can be dissipated in from thirty minutes to an hour and a half at that temperature. Since wax patterns are variable in size a good working rule is to keep the investment on a heat of between $300^{\circ}$ and $320^{\circ} \mathrm{F}$ for one and a half hours, when the wax will be dissipated and the case may be set off to cool.

Since it is essential to keep the tem- 
perature of the investment within rather close limits it is necessary to use something more than an open flame over which to heat the case. The playing of a flame directly upon an investment causes a rapid deterioration and distortion of the plaster of Paris. Most of the deleterious effect is at first confined to the immediate surface of the investment but there is no means of judging how rapidly it is penetrating, so the walls of the mould may be affected before the operator is aware of it.

The case should not be heated too rapidly. The rapid rising of an invested case to a high temperature causes the wax to melt, steam to form, and a series of peculiar wax explosions to take place in the mould. Continued intense heat finally causes the wax to boil. The agitation of the wax due to explosions and boiling works out the particles of silex, graphite, etc., which are not directly held by the binder, from the walls of the mould. A casting made in such a mould comes out with a roughly etched surface. If the heat is sufficient to disintegrate the plaster more particles of silex, etc., are dislodged and a rougher casting results. A rough casting cannot fit a cavity as perfectly as a smooth one. Furthermore too rapid heating may cause the investment to crack and a "feathered" inlay result.

Many devices have been used to assist in carrying out the principles outlined above. A heating arrangement that will best answer the purpose must provide (1) control of the heat, (2) registration of the temperature, (3) prevent the flame or heating unit from coming in direct contact with any part of the investment or ring and (4) a more or less even distribution of the heat all around the invested case. A small asbestos lined oven equipped with a vulcanizer stud and thermometer furnishes a satisfactory appliance. An electric unit or gas flame may be utilized to furnish the heat but a flash plate must be provided to distribute the heat evenly thruout the chamber and prevent the heating unit from coming in contact with the invested case. A vulcanizer can be used with satisfaction. An empty flask should be placed in the bottom of the bowl. The flame of the vulcanizer plays directly on the bottom of the bowl so that it becomes too hot for an investment to rest upon. The empty flask acts as a flash plate. Since no steam pressure is developed the cover of the vulcanizer need not be clamped. Dr. Hollenbeck. of Lewiston, Montana, has devised an electrically heated and controlled apparatus. It is unique and should give very satisfactory results.

If the reader is skeptical concerning the ideas expressed so far and does not care to invest in additional equipment, an empirical method that gives satisfactory results is as follows: Upon a small household gas plate a circle of stove pipe iron large enough to cover the top of the stove is placed. Another piece of the stove pipe metal or tin eight or ten inches high is bent to form a draft shield extending more than half way around the stove. Two or three old burs on the plate will serve to raise the invested case from the hot plate. If the experimenting is done at night or in a dark room the flame should be turned until small areas of dark red appear on the stove pipe metal. The position of the gas cock should be noted so that like results may be obtained at anytime. It requires from one to two hours to dissipate the wax in this comparatively open apparatus as the air currents of the room modify the temperature. In ten or fifteen minutes after the case is put on heat, steam will rise from it. Later smoke may appear from the wax that has run out of the sprue hole. At the end of an hour the ring is grasped with a pair of tongs and quickly tipped. If no further smoke immediately rises the 
case is considered done and set off to cool. If smoke immediately rises, on tipping, the heat is continued for fifteen or twenty minutes longer and the same test is repeated. On the completion of this method of dissipating the wax the crucible will be discolored. The discolorization will vary from a light grey to almost black. This last method is purely empirical and while it gives good results the writer does not feel that it is as accurate as one in which a heating apparatus with proper control and thermometer is used.

\section{Casting.}

Perfect castings are made only in moulds that have been permitted to cool to room temperature-the so-called "cold mould." A mould that is too hot to be borne comfortably on the back of the hand is a distorted mould and a distorted inlay is the result of casting in such a mould. The hotter the mould the more distortion is present. After the wax has been dissipated and the mould has cooled the casting may be done any time.

There has been much discussion concerning the karat of gold to be used in casting. Excellent casting can be made with $22 \mathrm{~K}, 23 \mathrm{~K}$ and $24 \mathrm{~K}$ gold when properly handled. Twenty-two karat gold has many qualities which make it a very desirable material for inlays in most cases. Many operators contend that only pure gold can be used to make accurately fitting inlays. They argue that pure gold has less contraction on cooling from a molten mass to a solidified state than any alloy of gold. Such statements are true. Up to the present time no rational technic has been advocated that will minimize this contraction. The gold should be heated only to a temperature that will permit it to be forced to place in the mould. The higher the temperature to which gold is brought the greater will be the contraction from the molten mass to the solidified state. Therefore, when a mould is filled with super-heated gold the contraction is in proportion to the superheat. It is contended that the contraction together with the maintained pressure used in casting will cause more gold from the surplus in the crucible to enter the mould and make up for shinkage. These contentions cannot be substantiated by practical demonstration and many valid reasons can be given to nullify the arguments. Inlays that have been cast with super-heated gold do not fit the cavity in their long diameters. Operators resort to excessive cavo-surface laps, not bevels, and advocate the burnishing of such laps to obtain what is nothing more than a cobbled fit. Such misfits are especially noticeable at the gingival of proximo-occlusal or still more in mesio-occluso-distal restorations. But a casting that is made with due regard to the technic of this paper will accurately fit a cavity which is prepared according to the principles laid down by Dr. Black. Such a cavity requires only a moderate beveling of the cavo-surface angle.

Many methods of forcing the gold into the crucible are employed. In view of the fact that it is desirable to avoid subjecting the investment to any more heat than is necessary some form of the centrifugal machine is the choice. The gold can be melted outside the crucible so that very little direct heat reaches the investment. Of the centrifugal machines, those activated by a smooth but quick acting spring seem most desirable, because of the almost instantaneous movement of the molten gold into the crucible upon releasing the spring. When gold is heated to a point only sufficiently molten to insure its adaptation to the walls of the mould this conservation of time in getting it to place is important.

Of the methods of casting that require the melting of the gold in the crucible, the simplest form is by use of a wet asbestos pad or "steam method" as it is 
named. The process is too familiar to need description here. Satisfactory inlays can be made by this method.

The writer has not carried on extensive experimentation with other machines. The principle objection is the necessity of melting the gold in the crucible and therefore the possible distortion of the mould. If any method that requires the melting of gold in the crucible is to be employed, thicker investments must be provided around the mould. Therefore, larger rings than those used in a centrifugal machine are clesirable. In melting gold in a crucible a small pointed flame should be used to concentrate the heat upon the gold. The gold should be melted as quickly as possible. It should be remembered that all moulds start as "cold moulds" in casting no matter what form of apparatus is used.

The question now arises as to what tests can be used to determine how hot the gold should be to make a desirable cast. The ocular test gained by experimenting is about the only guide. Upon first melting, gold tends to assume a spherical form. If heat is continued, it is soon observed that a film breaks on the surface of the sphere and retracts down over the sides, leaving a bright mirror-like surface. If the heat is decreased just an instant this film reappears, giving a dull lusterless coating. Increase the heat and the film again retracts. Gold is hot enough to cast in a centrifugal machine when it reaches the stage when it is covered by that mirror-like surface. It will be light yellow in color. In using steam or compressed air a little more temperature is needed. The gold will have that mirrorlike surface but should be a much lighter yellow in color. After the gold has been brought to the desired temperature it should be cast as quickly as possible. The reason is obvious.

The heating of the gold can be accomplished by many ways. In most localities the city gas is of a quality that answers the purpose if a suitable blow pipe is obtained. When gold is to be melted in the crucible, where rapidity of melting is desirable, acetylene, oxyhydrogen or some other combination may be advisable if the city gas is not exceptionally good.

\section{RECAPITULATION.}

(1) Wax forms should be mounted, washed with alcohol and invested as soon as possible after removal from the cavity.

(2) Wax forms, investment materials, and water to mix the latter should be kept at room temperature.

(3) As soon as the investment has set sufficiently to give a reasonable resistance to the finger nail the former and sprue should be removed and the case put on heat immediately, crucible end down.

(4) The temperature at which the wax is dissipated should not exceed $320^{\circ}$ $\mathrm{F}$ and should be continued one hour and one-half. The investment or the ring should not come in direct contact with the heating element.

(5) The casting should be done in a cold mould. The gold in casting should be raised to a temperature just sufficiently high to permit it to be forced to adaption to the walls of the mould.

(6) A machine that allows the melting of the gold outside the crucible of the investment is preferable. 